\title{
Population Dynamics of Exelastis atomosa (Walsingham) on Pigeonpea Genotypes in Agro-Ecosystem
}

\author{
Rahul Kumar*, Ram Keval and Vijay Kumar Mishra \\ Department of Entomology and Agricultural Zoology, Institute of Agricultural Sciences, Banaras \\ Hindu University, Varanasi-221005, Uttar Pradesh, India \\ *Corresponding author
}

\section{A B S T R A C T}

\begin{tabular}{l} 
K e y w o r d s \\
Pigeonpea crop, \\
$\begin{array}{l}\text { Exelastisn atomosa } \\
\text { (Walsingham), } \\
\text { Population } \\
\text { dynamics }\end{array}$ \\
Article Info \\
\hline $\begin{array}{l}\text { Accepted: } \\
\text { 25 March } 2017 \\
\text { Available Online: } \\
10 \text { April } 2017\end{array}$ \\
\hline
\end{tabular}

\section{Introduction}

India is one of the leading countries in pulse production as well as consumption. It accounts for 33 percent of world area and 24 percent of world production.The most important states for pulses production are Maharashtra, Uttar Pradesh, Madhya Pradesh, Andhra Pradesh, Karnataka, Gujarat and Bihar, which together account for more than 80 per cent of total pulses production. At present the production of pulses is 18.34 million tonnes over an area of 23.26 million hectares with productivity of $789 \mathrm{Kg} / \mathrm{ha}(\mathrm{E}$ Pulses data book IIPR, 2015). Pigeonpea production is affected by several biotic and abiotic stresses. Among biotic factors, the seeds and other parts of the plant are fed upon by many insects, with over 200 species having been recorded in India alone. Some of these insects cause sufficient crop losses to be regarded as major pests, but the majority are seldom abundant enough to cause much damage, or are of sporadic or localized importance, and regarded as minor pests. The pod-damaging insect (Plume moth) cause significant yield losses in pigeonpea and therefore are the most important pests of this crop. Pests that feed on reproductive structures, flowers, and pods cause the greatest harm. Foliar damage rarely reduces seed yield. The post-harvest insect pests are 
also considered significant in inflecting losses during storage. The plume moth in particular often assume greater significance and are recognized as the major constraints in productivity (Rangaiah and Sehgal, 1984). From different parts of the country occurrence of a complex of pod borers had been reported in pigeon pea (La1 et al., 1981; Khokhar and Singh, 1983; Upadhyay et al., 1998). For development of successful pest management strategies, detailed information on the population build up, in particular the influence of weather factors on the population dynamics is of great significance. The larva was the damaging stage, which feeds on buds, flowers and young pods and as a result of feeding, small holes are seen on the buds and tender pods. Plume moth infested ripened pods show characteristic fungal attacked grains which were brittle, blackened and unfit for consumption. These findings are in accordance with the findings of Srilaxmi and Ravinda (2010); Subharani and Singh (2004) and Yadav et al. (2009 .The present study focuses on the information on the population build up and its influence with different genotypes of pigeonpea, on which available knowledge is scanty. This formulation are more important in of the pest management strategy based on host plant resistance

\section{Materials and Methods}

The studies were carried out at Agricultural Research Farm, Institute of Agricultural Sciences, Banaras Hindu University, Varanasi, during Kharif, 2013-2014 and 2014-2015. The Eighteen long duration pigeonpea genotypes/varieties were grown in plots of 5 rows of 4 meters following row to row and plant to plant spacing of $75 \mathrm{~cm}$ and $15 \mathrm{~cm}$ respectively. The crop was grown following the normal agronomic practices in "Randomized Block Design" with three replications and eighteen treatments. The crop was shown on 26th July during 2013-14 and 1st August during 2014-15 and harvested on 7th April 2014 and 10th April 2015 respectively. The whole plot was exposed to natural infestation and no insecticides applied.For recording the seasonal incidence of insect pest, five plants were randomly selected in each treatment and tagged. The immature stages of pod borers present on them were counted at weekly intervals, from 24th January to 28st march during 2013-14 and 2014-15. This practice was continued throughout the pod formation stage. The weekly observation on the number of larvae and pupae in the sampled pods during both the years was taken from 24th January to $28^{\text {th }}$ march. The number of insect count recorded from all the three replication for all the genotypes were average separately for each genotype on standard week basis from all the replication of 18 genotypes/varieties of pigeon pea.

\section{Statistical analysis}

All the data recorded were subjected to statistical analysis as per the Randomized Block Design procedure and insect population data were transformed with square root transformed $\sqrt{ } \mathrm{x}+0.5$ method.

\section{Results and Discussion}

\section{Population dynamics of plume moth, Exelastis atomosa (Walsingham)}

During 2013-14 the first incidence of Plume moth, Exelastis atomosa was observed in $4^{\text {th }}$ standard week in nine genotypes i.e. DA 13-2, MAL 40, MA 6, NDA 13-1, NDA 13-1, MAL 13, IPA 7-10, DA 13-1, BHUA 189. The rest allthe genotypes noticed pest incidence in $5^{\text {th }}$ standard week except KA 12-2 and KA 12-4 in which first incidence recorded on $6^{\text {th }}$ standard week. The peak of population of Plume moth was recorded in different genotype from $11^{\text {th }}$ to $12^{\text {th }}$ standard week. 
Table.1 Plume moth (Exelastis atomosa (Walsingham) population on long duration pigeonpea genotypes during Kharif 2013-14

\begin{tabular}{|c|c|c|c|c|c|c|c|c|c|c|c|}
\hline \multirow[b]{2}{*}{ Genotypes } & \multicolumn{10}{|c|}{ Population per plant } & \multirow[b]{2}{*}{ Average } \\
\hline & $\begin{array}{l}22^{\text {th }} \\
\text { Jan }\end{array}$ & $\begin{array}{l}29^{\text {th }} \\
\text { Jan }\end{array}$ & $\begin{array}{c}5^{\text {th }} \\
\text { Feb }\end{array}$ & $\begin{array}{l}12^{\text {th }} \\
\text { Feb }\end{array}$ & $\begin{array}{l}19^{\text {th }} \\
\text { Feb }\end{array}$ & $\begin{array}{l}26^{\text {th }} \\
\text { Feb }\end{array}$ & $\begin{array}{c}5^{\text {th }} \\
\text { March }\end{array}$ & $\begin{array}{c}{12^{\text {th }}} \\
\text { March }\end{array}$ & $\begin{array}{c}19^{\text {th }} \\
\text { March }\end{array}$ & $\begin{array}{c}2^{\text {th }} \\
\text { March }\end{array}$ & \\
\hline DA 13-2 & $0.07(1.03)$ & $0.15(1.07)$ & $0.18(1.09)$ & $0.27(1.13)$ & $0.34(1.16)$ & $0.39(1.18)$ & $0.52(1.23)$ & $0.60(1.26)$ & $0.61(1.27)$ & $0.16(1.08)$ & 0.33 \\
\hline MAL 40 & $0.04(1.02)$ & $0.07(1.03)$ & $0.13(1.06)$ & $0.19(1.09)$ & $0.25(1.12)$ & $0.33(1.15)$ & $0.43(1.20)$ & $0.63(1.27)$ & $0.52(1.23)$ & $0.15(1.07)$ & 0.27 \\
\hline BAHAR(ch) & $0.00(1.00)$ & $0.03(1.02)$ & $0.07(1.03)$ & $0.14(1.07)$ & $0.19(1.09)$ & $0.22(1.10)$ & $0.37(1.17)$ & $0.49(1.22)$ & $0.44(1.20)$ & $0.07(1.03)$ & 0.20 \\
\hline MA 6 (ch) & $0.12(1.06)$ & $0.20(1.10)$ & $0.28(1.13)$ & $0.38(1.18)$ & $0.49(1.22)$ & $0.57(1.25)$ & $0.81(1.35)$ & $0.99(1.41)$ & $0.89(1.37)$ & $0.3(1.14)$ & 0.50 \\
\hline IPA 11-1 & $0.00(1.00)$ & $0.13(1.06)$ & $0.18(1.09)$ & $0.20(1.10)$ & $0.30(1.14)$ & $0.35(1.16)$ & $0.50(1.22)$ & $0.69(1.30)$ & $0.70(1.30)$ & $0.19(1.09)$ & 0.32 \\
\hline NDA 13-1 & $0.10(1.05)$ & $0.15(1.07)$ & $0.23(1.11)$ & $0.35(1.16)$ & $0.45(1.20)$ & $0.53(1.24)$ & $0.78(1.33)$ & $0.88(1.37)$ & $0.79(1.34)$ & $0.24(1.11)$ & 0.45 \\
\hline KA 12-2 & $0.00(1.00)$ & $0.00(1.00)$ & $0.04(1.02)$ & $0.12(1.06)$ & $0.13(1.06)$ & $0.20(1.10)$ & $0.35(1.16)$ & $0.45(1.20)$ & $0.37(1.17)$ & $0.06(1.03)$ & 0.17 \\
\hline NDA 1 (ch) & $0.05(1.03)$ & $0.12(1.06)$ & $0.15(1.07)$ & $0.21(1.10)$ & $0.27(1.13)$ & $0.34(1.16)$ & $0.47(1.21)$ & $0.65(1.28)$ & $0.57(1.25)$ & $0.19(1.09)$ & 0.30 \\
\hline MAL 13(ch) & $0.1(1.05)$ & $0.07(1.03)$ & $0.3(1.14)$ & $0.34(1.16)$ & $0.42(1.19)$ & $0.53(1.24)$ & $0.68(1.30)$ & $0.67(1.29)$ & $0.75(1.32)$ & $0.23(1.11)$ & 0.41 \\
\hline IPA 7-10 & $0.18(1.09)$ & $0.13(1.06)$ & $0.28(1.13)$ & $0.42(1.19)$ & $0.53(1.24)$ & $0.65(1.28)$ & $0.87(1.37)$ & $1.08(1.44)$ & $0.93(1.39)$ & $0.32(1.15)$ & 0.54 \\
\hline DA 13-1 & $0.07(1.03)$ & $0.18(1.09)$ & $0.15(1.07)$ & $0.24(1.11)$ & $0.21(1.10)$ & $0.43(1.20)$ & $0.67(1.29)$ & $0.74(1.32)$ & $0.59(1.26)$ & $0.16(1.08)$ & 0.34 \\
\hline NDA 13-2 & $0.00(1.00)$ & $0.08(1.04)$ & $0.13(1.06)$ & $0.18(1.09)$ & $0.24(1.11)$ & $0.35(1.16)$ & $0.55(1.24)$ & $0.59(1.26)$ & $0.52(1.23)$ & $0.14(1.07)$ & 0.28 \\
\hline BAUPP 09-22 & $0.00(1.00)$ & $0.01(1.00)$ & $0.10(1.05)$ & $0.15(1.07)$ & $0.20(1.10)$ & $0.27(1.13)$ & $0.39(1.18)$ & $0.50(1.22)$ & $0.45(1.20)$ & $0.09(1.04)$ & 0.22 \\
\hline MAL 39 & $0.00(1.00)$ & $0.08(1.04)$ & $0.12(1.07)$ & $0.17(1.08)$ & $0.25(1.12)$ & $0.31(1.14)$ & $0.45(1.20)$ & $0.64(1.28)$ & $0.56(1.25)$ & $0.13(1.06)$ & 0.27 \\
\hline KA 12-4 & $0.00(1.00)$ & $0.05(1.03)$ & $0.12(1.07)$ & $0.16(1.08)$ & $0.20(1.10)$ & $0.29(1.13)$ & $0.39(1.18)$ & $0.53(1.24)$ & $0.50(1.22)$ & $0.05(1.03)$ & 0.23 \\
\hline KA 12-3 & $0.00(1.00)$ & $0.00(1.00)$ & $0.05(1.03)$ & $0.14(1.07)$ & $0.18(1.09)$ & $0.20(1.10)$ & $0.36(1.16)$ & $0.47(1.21)$ & $0.39(1.18)$ & $0.07(1.03)$ & 0.19 \\
\hline NDA 2 (ch) & $0.00(1.00)$ & $0.07(1.03)$ & $0.10(1.05)$ & $0.19(1.09)$ & $0.22(1.10)$ & $0.31(1.14)$ & $0.43(1.20)$ & $0.58(1.26)$ & $0.51(1.23)$ & $0.11(1.05)$ & 0.25 \\
\hline BHUA 189 & $0.07(1.03)$ & $0.19(1.09)$ & $0.15(1.07)$ & $0.32(1.15)$ & $0.29(1.14)$ & $0.45(1.20)$ & $0.57(1.25)$ & $0.76(1.33)$ & $0.60(1.26)$ & $0.21(1.10)$ & 0.36 \\
\hline Average & 0.04 & 0.10 & 0.15 & 0.23 & 0.29 & 0.37 & 0.53 & 0.66 & 0.59 & 0.16 & - \\
\hline SEM \pm & 0.007 & 0.011 & 0.013 & 0.018 & 0.024 & 0.031 & 0.032 & 0.040 & 0.041 & 0.019 & - \\
\hline CD at $5 \%$ & 0.020 & 0.031 & 0.037 & 0.052 & 0.070 & 0.089 & 0.093 & 0.115 & 0.119 & 0.055 & - \\
\hline
\end{tabular}

Figures in parentheses are $\sqrt{\mathrm{x}}+0.5$ transformed value 
Table.2 Plume moth (Exelastis atomosa (Walsingham) population on long duration pigeonpea genotypes during Kharif2014-15

\begin{tabular}{|c|c|c|c|c|c|c|c|c|c|c|c|}
\hline \multirow[b]{2}{*}{ Genotypes } & \multicolumn{10}{|c|}{ Population per plant } & \multirow[b]{2}{*}{ Average } \\
\hline & $\begin{array}{l}22^{\text {th }} \\
\text { Jan }\end{array}$ & $\begin{array}{l}29^{\text {th }} \\
\text { Jan }\end{array}$ & $\begin{array}{c}5^{\text {th }} \\
\text { Feb }\end{array}$ & $\begin{array}{l}12^{\text {th }} \\
\text { Feb }\end{array}$ & $\begin{array}{l}19^{\text {th }} \\
\text { Feb }\end{array}$ & $\begin{array}{l}26^{\text {th }} \\
\text { Feb }\end{array}$ & $\begin{array}{c}5^{\text {th }} \\
\text { March }\end{array}$ & $\begin{array}{c}12^{\text {th }} \\
\text { March }\end{array}$ & $\begin{array}{c}19^{\text {th }} \\
\text { March }\end{array}$ & $\begin{array}{c}26^{\text {th }} \\
\text { March }\end{array}$ & \\
\hline DA 13-2 & $0.12(1.06)$ & $0.21(1.10)$ & $0.15(1.07)$ & $0.20(1.10)$ & $0.32(1.15)$ & $0.38(1.17)$ & $0.47(1.21)$ & $0.62(1.27)$ & $0.65(1.28)$ & $0.27(1.13)$ & 0.34 \\
\hline MAL 40 & $0.09(1.04)$ & $0.12(1.06)$ & $0.12(1.06)$ & $0.18(1.09)$ & $0.22(1.10)$ & $0.32(1.15)$ & $0.38(1.17)$ & $0.55(1.25)$ & $0.62(1.27)$ & $0.21(1.10)$ & 0.28 \\
\hline BAHAR(ch) & $0.00(1.00)$ & $0.05(1.03)$ & $0.07(1.03)$ & $0.12(1.06)$ & $0.15(1.07)$ & $0.20(1.10)$ & $0.33(1.15)$ & $0.41(1.19)$ & $0.49(1.22)$ & $0.15(1.07)$ & 0.20 \\
\hline MA 6 (ch) & $0.23(1.11)$ & $0.27(1.13)$ & $0.21(1.10)$ & $0.35(1.16)$ & $0.45(1.20)$ & $0.52(1.23)$ & $0.77(1.33)$ & $0.38(1.35)$ & $0.89(1.37)$ & $0.34(1.16)$ & 0.44 \\
\hline IPA 11-1 & $0.11(1.05)$ & $0.15(1.07)$ & $0.09(1.04)$ & $0.17(1.08)$ & $0.27(1.13)$ & $0.35(1.16)$ & $0.52(1.23)$ & $0.59(1.26)$ & $0.66(1.29)$ & $0.22(1.10)$ & 0.31 \\
\hline NDA 13-1 & $0.18(1.09)$ & $0.22(1.10)$ & $0.25(1.12)$ & $0.32(1.15)$ & $0.40(1.18)$ & $0.31(1.14)$ & $0.73(1.31)$ & $0.89(1.37)$ & $0.88(1.37)$ & $0.3(1.14)$ & 0.45 \\
\hline KA 12-2 & $0.00(1.00)$ & $0.05(1.03)$ & $0.00(1.00)$ & $0.09(1.04)$ & $0.11(1.05)$ & $0.17(1.08)$ & $0.27(1.13)$ & $0.35(1.16)$ & $0.42(1.19)$ & $0.00(1.0)$ & 0.15 \\
\hline NDA 1 (ch) & $0.05(1.03)$ & $0.13(1.06)$ & $0.12(1.06)$ & $0.22(1.10)$ & $0.25(1.12)$ & $0.31(1.14)$ & $0.60(1.26)$ & $0.59(1.26)$ & $0.66(1.29)$ & $0.23(1.11)$ & 0.32 \\
\hline MAL 13(ch) & $0.13(1.06)$ & $0.10(1.05)$ & $0.28(1.13)$ & $0.25(1.07)$ & $0.38(1.18)$ & $0.49(1.22)$ & $0.58(1.26)$ & () $0.72(1.31)$ & $0.82(1.35)$ & $0.29(1.14)$ & 0.40 \\
\hline IPA 7-10 & $0.20(1.10)$ & $0.39(1.15)$ & $0.30(1.14)$ & $0.39(1.18)$ & $0.49(1.22)$ & $0.60(1.27)$ & $0.81(1.34)$ & $0.97(1.40)$ & $0.98(1.41)$ & $0.36(1.17)$ & 0.55 \\
\hline DA 13-1 & $0.14(1.07)$ & $0.17(1.08)$ & $0.13(1.06)$ & $0.33(1.15)$ & $0.18(1.09)$ & $0.42(1.19)$ & $0.49(1.22)$ & $0.65(1.28)$ & $0.63(1.28)$ & $0.23(1.11)$ & 0.34 \\
\hline NDA 13-2 & $0.00(1.0)$ & $0.13(1.06)$ & $0.12(1.06)$ & $0.15(1.07)$ & $0.24(1.11)$ & $0.30(1.14)$ & $0.41(1.19)$ & $0.56(1.25)$ & $0.59(1.26)$ & $0.20(1.10)$ & 0.27 \\
\hline BAUPP 09-22 & $0.00(1.0)$ & $0.05(1.03)$ & $0.00(1.0)$ & $0.13(1.06)$ & $0.17(1.08)$ & $0.23(1.11)$ & $0.34(1.16)$ & $0.46(1.21)$ & $0.50(1.23)$ & $0.00(1.00)$ & 0.19 \\
\hline MAL 39 & $0.07(1.03)$ & $0.07(1.04)$ & $0.09(1.04)$ & $0.16(1.08)$ & $0.21(1.10)$ & $0.29(1.14)$ & $0.37(1.17)$ & $0.54(1.24)$ & $0.57(1.25)$ & $0.18(1.09)$ & 0.26 \\
\hline KA 12-4 & $0.00(1.0)$ & $0.09(1.04)$ & $0.00(1.0)$ & $0.15(1.07)$ & $0.20(1.10)$ & $0.27(1.13)$ & $0.39(1.18)$ & $0.42(1.19)$ & $0.52(1.23)$ & $0.00(1.00)$ & 0.20 \\
\hline KA 12-3 & $0.00(1.0)$ & $0.07(1.04)$ & $0.00(1.0)$ & $0.11(1.05)$ & $0.15(1.07)$ & $0.17(1.08)$ & $0.29(1.14)$ & $0.37(1.17)$ & $0.43(1.19)$ & $0.00(1.00)$ & 0.16 \\
\hline NDA 2 (ch) & $0.00(1.0)$ & $0.11(1.05)$ & $0.10(1.05)$ & $0.13(1.06)$ & $0.17(1.08)$ & $0.26(1.12)$ & $0.34(1.16)$ & $0.49(1.22)$ & $0.55(1.24)$ & $0.18(1.09)$ & 0.23 \\
\hline BHUA 189 & $0.15(1.07)$ & $0.19(1.09)$ & $0.15(1.07)$ & $0.28(1.13)$ & $0.18(1.09)$ & $0.48(1.22)$ & $0.45(1.20)$ & $0.69(1.30)$ & $0.74(1.31)$ & $0.12(1.06)$ & 0.34 \\
\hline Average & 0.08 & 0.14 & 0.12 & 0.20 & 0.25 & 0.34 & 0.47 & 0.59 & 0.64 & 0.17 & - \\
\hline SEM \pm & 0.007 & 0.010 & 0.010 & 0.016 & 0.015 & 0.020 & 0.034 & 0.030 & 0.033 & 0.017 & - \\
\hline CD at $5 \%$ & 0.021 & 0.029 & 0.029 & 0.048 & 0.044 & 0.057 & 0.098 & 0.088 & 0.095 & 0.050 & - \\
\hline
\end{tabular}


Fig.1 Population fluctuation of Plume moth on different long duration pigeonpea genotypes/varieties

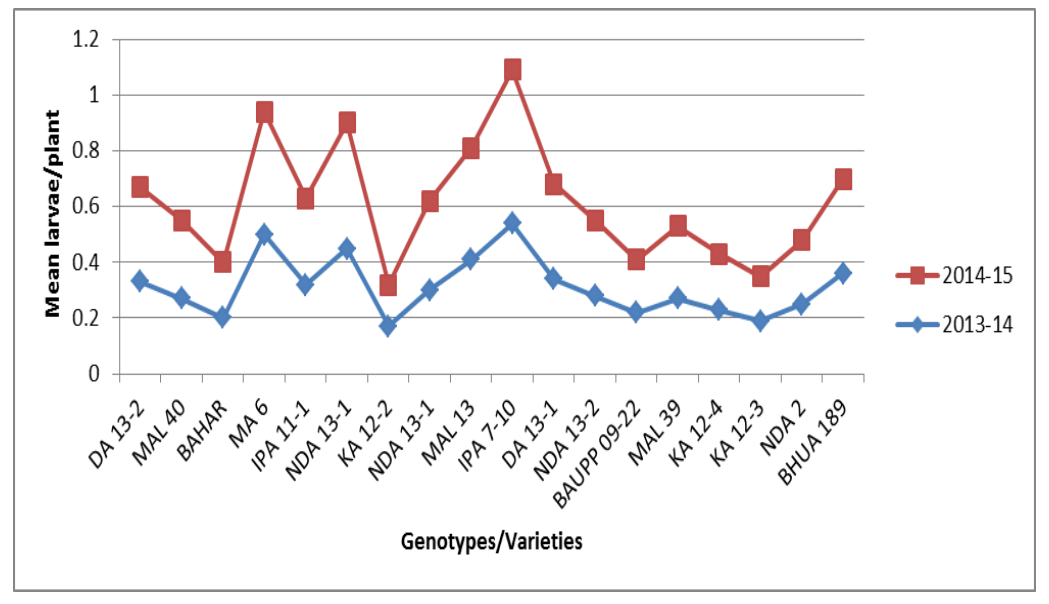

Fig.2 Seasonal incidence of Plume moth on long duration pigeonpea

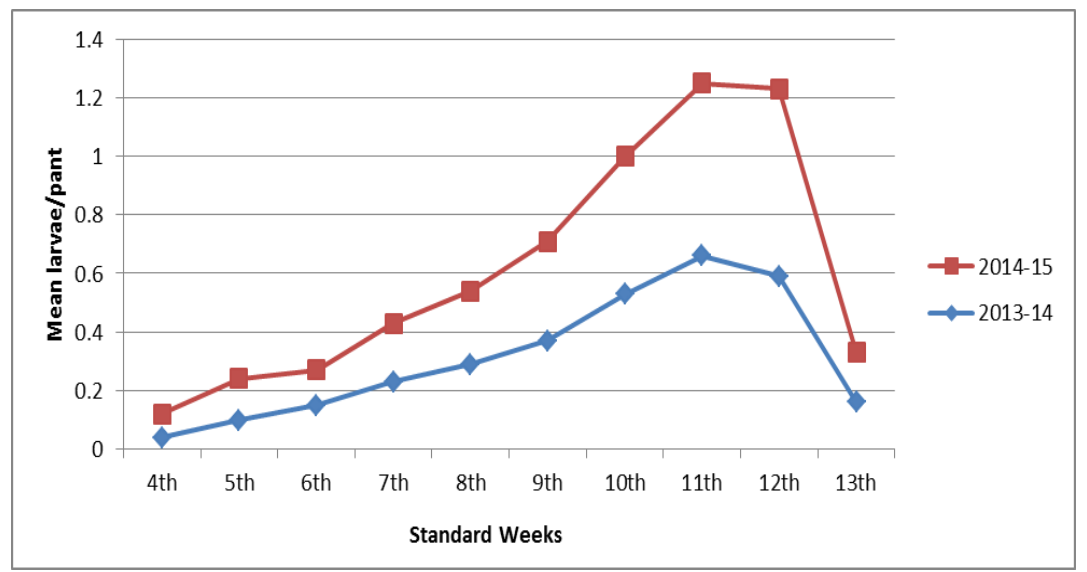

The peak population of plum moth was observed on $11^{\text {th }}$ standard week except DA 13-2, IPA 11-1 $\&$ NDA 1 in which peak observed on $12^{\text {th }}$ standard week.

Among the eighteen genotypes/varieties, the mean population of Plume moth was recorded highest in genotype IPA 7-10 i.e. (0.54 larvae/plant) followed by MA-6 (0.50 larvae/plant), NDA 13-1 (0.45 larvae/plant), and lowest in genotype i.e. KA 12-2 (0.17 larvae/plant) followed by KA 12-3 (0.19 larvae/plant), BAHAR (0.20 larvae/plant).The mean population of plum moth was recorded highest in $11^{\text {th }}$ standard week i.e. 0.66 larvae/plant followed by $12^{\text {th }}$ standard week (0.59 larvae/plant) and lowest population was recorded in $4^{\text {th }}$ standard week i.e. $(0.04$ larvae/plant).
During 2014-15 the first incidence of Plume moth, Exelastis atomosa was observed on $4^{\text {th }}$ standard week in all genotypes except BAHAR, KA 12-2, NDA 13-2, BAUPP 09-22, KA 12-3, NDA 2 in which first incidence observed on $5^{\text {th }}$ standard week. The peak of population of Plume moth was recorded in different genotype from $11^{\text {th }}$ to $12^{\text {th }}$ standard week. The peak population of plum moth was observed on $12^{\text {th }}$ standard week on all genotypes except DA 13-1 \& NDA 13-1 in which peak observed on $11^{\text {th }}$ standard week.Among the eighteen genotypes/varieties, the mean population of Plume moth was recorded highest in genotype IPA 7-10 i.e. (0.55 larvae/plant) followed by NDA 13-1 (0.45 larvae/plant) MA-6 (0.44 larvae/plant), and lowest in genotype i.e. KA 12-2 (0.15 larvae/plant) followed by KA 12-3 (0.16 larvae/plant), BAHAR (0.20 larvae/plant).The 
mean population of plum moth was recorded highest in $12^{\text {th }}$ standard week i.e. 0.64 larvae/plant followed by $11^{\text {th }}$ standard week (0.59 larvae/plant) and lowest population was recorded in $4^{\text {th }}$ standard week i.e. (0.08 larvae/plant).Present investigation related with,Senapatiet al. (2000), who recorded its incidence from middle of October to middle of February with peaks in fourth week of October, third and fourth weeks of January on early, medium and late duration varieties, respectively, in Orissa.Kumar and Nath (2005)reported the average population of Exelastis atomosa 0.92 plants.Chavanet al., (2010)conducted field studies to determine the resistance of 11 pigeonpea genotypes against Exelastisatomosa. Pawar et al.,(2014) was reported that First appearance of the red gram plume moth larva was observed when the crop age was about 130 days, 47th SW,From the evident that the pest was present on the crop during the reproductive stage and remained available upto the first week of January, $1^{\text {st }}$ SW i.e. maturity stage of the crop (172 CAD).

\section{References}

Annonymou,2015.http://www.commoditiescontro 1.com/eagritrader/commodityknowldege/r edgram/redgram.1.htm\#, accessed .

Chavan, A.P., Bramhane, R.O., Shinde, V.B. andHarer, P.N. 2010. Resistance against pod borer complex in.Journal of Maharashtra Agricultural Universities, 35(3): 469-470

Khokhar. K.S. and Singh, Z. 1983. Insect pests associated with pigeon pea at Hisar, India international pigeonpea newsletter, 3: 43 -45 .

Kumar, A. and Nath, P. 2003. Pest complex and their population dynamics on medium-late variety ofBahar. Indian Journal ofPulses Research, 16(2): 150-154.
Lal, S.S ,Yadava, C.P and Das, C. A.R. 1981.Major pest problems of pigeon pea In U P, lndialnternational Plgeonpea Newes letter. 1: 30 -31.

Pawar,U.A., Chintkuntalawar, P.S., AND Ugale, T.B. 2014. Studies on succession of insect pest complex and their natural enemies in pigeonpea [Cajanus cajan (L.) Millsp. International Journal of Plant Protection.,7 (2): 318-324.

Rangalah, P.V. and Sehgal, V.K. 1984. Estlmation of crop losses in pigeon pea due to Insect pests associated with in various stages of its growth. Indian Journal of Plant Breeding, 12: 127 -130.

Senapati, B.,Sahoo, B.K.,Kulat. S.S. and Bodhade, S.N. 2000.Insect pests of pigeonpea and their management.ApplledEntoomolog, insect pests of pulses \& oilseeds and their manngemen. 2, 28- 53.

Srilaxmi, K. and Ravinda P. 2010. Diversity of insect pests of pigeonpea [Cajanus cajan (L.) Millsp.] and their succession in relation to crop phenology in Gulbarga, Karnataka. The Ecoscan., 4(4): 273-27.

Subharani, S. and Singh, T.K. 2004. Insect pest complex of pigeonpea (Cajanus Cajan) in agro-ecosystem of Manipur. Indian $\mathrm{J}$. Entomol., 66(3): 222-224.

Upadhyay, R.K. Mukherji, K.G. and Rajak, R.L. 1998. IPM system in agriculture , 4 pulses, New Delhi. 99 pp

Yadav, R.S., Rai, S.N., Prasad, S. and Sing, J. 2009. Diversity of insect pests in pigeonpea, Cajanus cajan (L.) Millsp, in eastern Uttar Pradesh. Appl. Zool. Res., 20(1): 127-131

\section{How to cite this article:}

Rahul Kumar, Ram Keval and Vijay Kumar Mishra. 2017. Population Dynamics of Exelastis atomosa (Walsingham) on Pigeonpea Genotypes in Agro-Ecosystem. Int.J.Curr.Microbiol.App.Sci. 6(4): 26292634. doi: https://doi.org/10.20546/ijemas.2017.604.306 